\title{
Aplicación del deep learning para el reconocimiento facial con la presencia de oclusiones en el contexto de la pandemia covid 2021
}

\author{
Jemima Elias Mucha, Angiela Rojas Ortega, Luis M. Rojas Salazar, Milagros M. Alfaro Montero, Roy C. \\ Uscamayta Pahuacho, Abraham E. Gamarra Moreno \\ Universidad Nacional del Centro del Perú, Mariscal Castilla 3909, Huancayo, Perú \\ Recibido el 15 de junio del 2021. Aceptado el 28 de junio del 2021.
}

DOI: https://doi.org/10.33017/RevECIPeru2021.0002/

Resumen

El Deep learning permite que una máquina aprenda de manera autónoma utilizando redes neuronales. Este artículo aplica el Deep learning para el reconocimiento facial inclusive cuando se utiliza mascarillas y/o lentes. La investigación usa Python y la librería de la red convolucional pre entrenada VGG16. El entorno de aplicación requiere un dataset donde se almacena las fotografías de los participantes, que ascienden a un total de 2400 imágenes, capturando en esencia la amplitud del rostro utilizando mascarilla y/o lentes; y colocadas en tres subcarpetas: Train, Test y Valid. En una primera fase se realiza el entrenamiento, dándose el aprendizaje en 500 epochs, y que al finalizar se obtiene la red entrenada. En una segunda fase se realiza el reconocimiento facial con la presencia de oclusiones en el rostro; utilizando una cámara web. El accuracy o precisión alcanzada en el entrenamiento de la red neuronal en Google colab es de 0.2 y el porcentaje de acierto obtenido de la aplicación en la segunda fase es de $71 \%$. El proyecto logra detectar a personas cuando estas utilizan mascarilla, lentes, ambos o sin el uso de estos; con el porcentaje de acierto mencionado anteriormente.

Descriptores: Deep Learning, Inteligencia Artificial, Redes Neuronales, Reconocimiento facial, VGG16.

\begin{abstract}
Deep learning allows a machine to learn autonomously using neural networks. This article applies Deep learning for facial recognition even when using masks and / or glasses. The research uses Python and the pre-trained convolutional network library VGG16. The application environment requires a dataset where the photographs of the participants are stored, which amount to a total of 2400 images, essentially capturing the breadth of the face using a mask and / or lenses; and placed in three subfolders: Train, Test and Valid. In the first phase, the training is carried out, learning in 500 epochs, and that at the end the trained network is obtained. In a second phase, facial recognition is performed with the presence of occlusions on the face; using a webcam. The accuracy or precision achieved in the training of the neural network in Google Collab is 0.2 and the percentage of success obtained from the application in the second phase is $71 \%$. The project manages to detect people when they use a mask, glasses, mask and glasses or without the use of these; with the success rate mentioned above.
\end{abstract}

Keywords: Deep Learning, Artificial Intelligence, Neural Networks, Facial recognition, VGG16. 


\section{Introducción}

En los últimos años, el sistema de reconocimiento facial fue desarrollado rápidamente para usos dentro de seguridad informática, seguridad pública, economía civil y entretenimiento [1], de igual manera en el registro de asistencia a los trabajos, como opción segura [2]. Sin embargo, en el avance de la tecnología se encuentran diversos desafíos para el reconocimiento facial. Dentro de los desafíos se encuentran situaciones como la variación de la iluminación que causa cambios dramáticos en los rasgos faciales [3]. El proceso de reconocimiento facial contempla la detección de objetos faciales, extracción de rasgos faciales y proceso de clasificación [4], durante la extracción de rasgos faciales se incluye desafíos como: variación facial, cambios en las expresiones faciales, cambio de escala, efecto de desenfoque y presencia de oclusiones, cuando se utiliza accesorios como lentes, chalinas, gorras, etc., que causan perdida de información importante [5].

El uso de un accesorio no permite un buen reconocimiento facial, ya que es considerado una oclusión; pero hay casos en que no se puede evitar el uso de los lentes, por el problema visual que tiene una persona; tampoco se puede evitar el uso de una mascarilla a causa de la situación actual, con la llegada del COVID-19. Además, la OMS declaro al COVID-19 como una pandemia el 2020 por su gravedad y alto nivel de propagación [6], donde los gobiernos tomaron medidas de emergencia para proteger a sus ciudadanos. En el Perú se dispuso el uso obligatorio de la doble mascarilla como medida de prevención para la circulación en la vía pública, así como en el ingreso a los supermercados y centros laborales, según el decreto Supremo №3-2021-PCM [7].

La Inteligencia Artificial (IA) se encarga de estudiar modelos capaces de realizar actividades propias de los seres humanos en base al razonamiento y conducta [8]. Dentro de la IA se encuentran dos ramas más específicas, el Machine Learning (ML) referido al aprendizaje automático que procura crear programas para generalizar comportamientos a partir de una información [9]. La rama del Deep Learning (DL) imita el funcionamiento del cerebro humano al procesar datos y crear patrones, compuesto por redes capaces de aprender de datos no estructurados [9].

El DL tiene como principal ventaja la posibilidad de usar una cantidad robusta de muestras para el entrenamiento, permitiendo aprender la representación facial resiliente a cambios en los datos de entrenamiento [10]. Para lo cual, si se desea obtener mayor precisión, se requiere ampliar el dataset y familiarizar a los sistemas con las características de cada rostro [11]. Lo que se busca es disminuir la cantidad de características inútiles de los datos faciales para buscar las características únicas y efectivas para la clasificación [12].

El articulo aplica las herramientas y proceso mencionado para lograr un reconocimiento facial efectivo, aun cuando el sujeto de estudio cuenta con los accesorios de mascarilla y lentes que causan oclusiones dentro del proceso de reconocimiento que afectan el resultado en la identificación confundiendo a los sujetos de prueba.

El Dataset utilizado es de 480 imágenes en diferentes ángulos y con diferentes accesorios que podrían crear oclusiones de cinco personas, formando subcarpetas, que son: Train, Valid y Test de 400, 40 y 40 imágenes respectivamente para el entrenamiento, validación y prueba, contando con un total de 2400 imágenes.

Usando las imágenes ingresadas en el dataset de los sujetos de prueba, se empieza la fase de entrenamiento para generar conocimiento, que una vez finalizado será conocido como la red neuronal entrenada. Usando esta red podremos identificar en base al patrón de imágenes a los sujetos de prueba.

El articulo realiza el reconocimiento facial en los siguientes casos. 1) con el rostro descubierto, 2) Con el uso de mascarilla quirúrgica, 3) con el uso de lentes, y 4) con el uso de mascarilla quirúrgica y lentes. El entrenamiento se dio 500 epochs durante 8 horas, muestra una precisión del 0.2 de error en el reconocimiento, que realizando las pruebas se obtuvo un $71 \%$ de acierto.

\section{Metodología}


En el desarrollo del artículo, se da en dos fases: la ejecución (etapa 3 y 4), tal como se muestra en la fase de entrenamiento (etapa 1 y 2 ) y la fase de Figura 1.

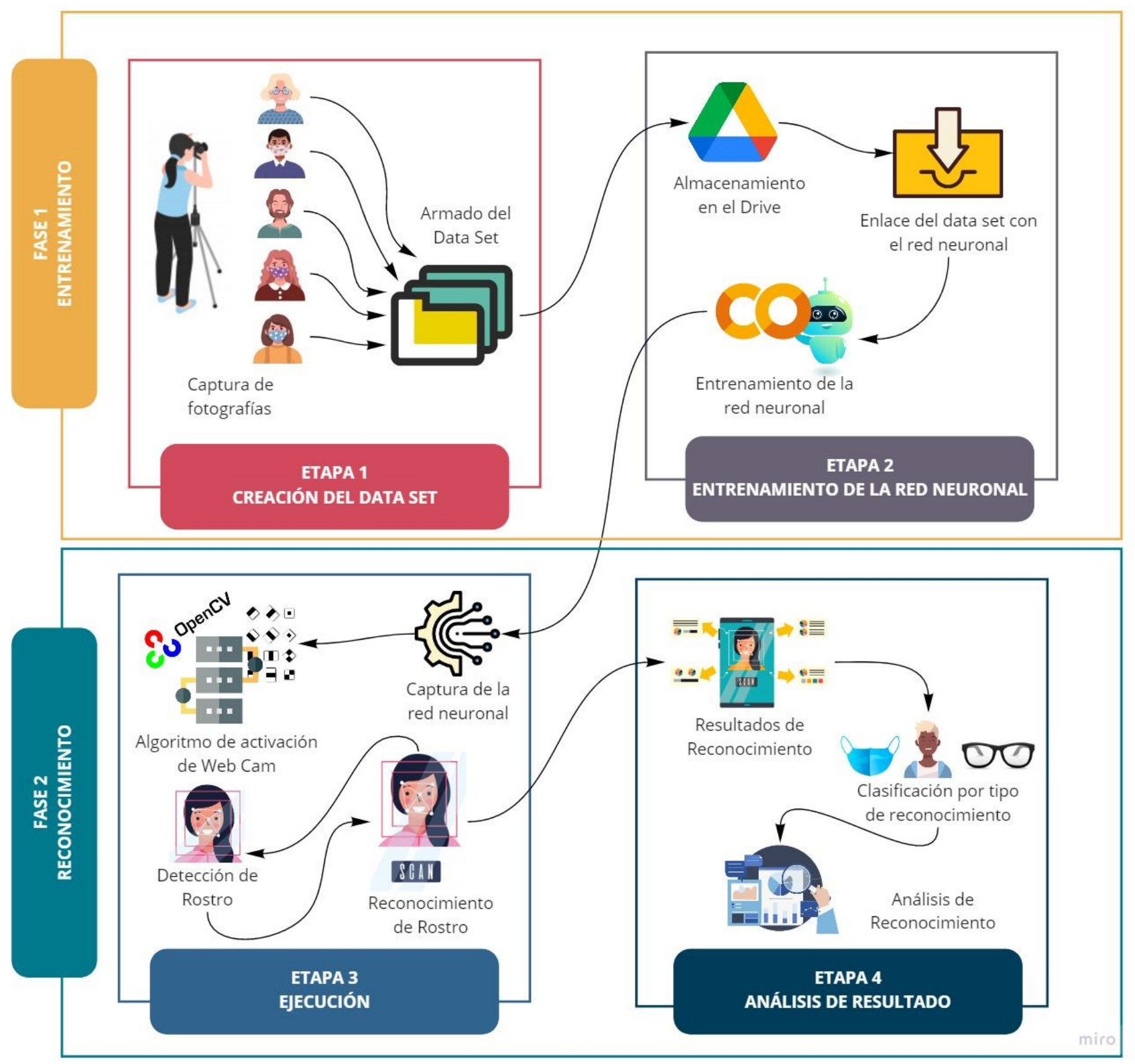

Figura 1: Fases y etapas de la metodología

\subsection{Etapa 1: creación del dataset}

Para la creación del dataset hubo un total de 2000 fotografías a colores como muestra, recortadas manualmente, almacenadas como se muestra en la 
Figura 2. Dentro de cada carpeta personal se tiene fotografías enfocado al rostro en diferentes ángulos de la persona sin movimiento corporal sin perder los rasgos que caracteriza a cada persona, como los ojos, nariz y boca, contando con accesorios como mascarillas y lentes.

\subsection{Etapa 2: entrenamiento de la red neuronal}

Para el entrenamiento de la red neuronal es necesario que se cargue el dataset comprimido a la nube, puesto que las imágenes recolectadas son en gran cantidad y llega a pesar demasiado, lidiar con ello es subir todas las imágenes a la nube. El alojamiento utilizado para el almacenamiento del dataset es google drive

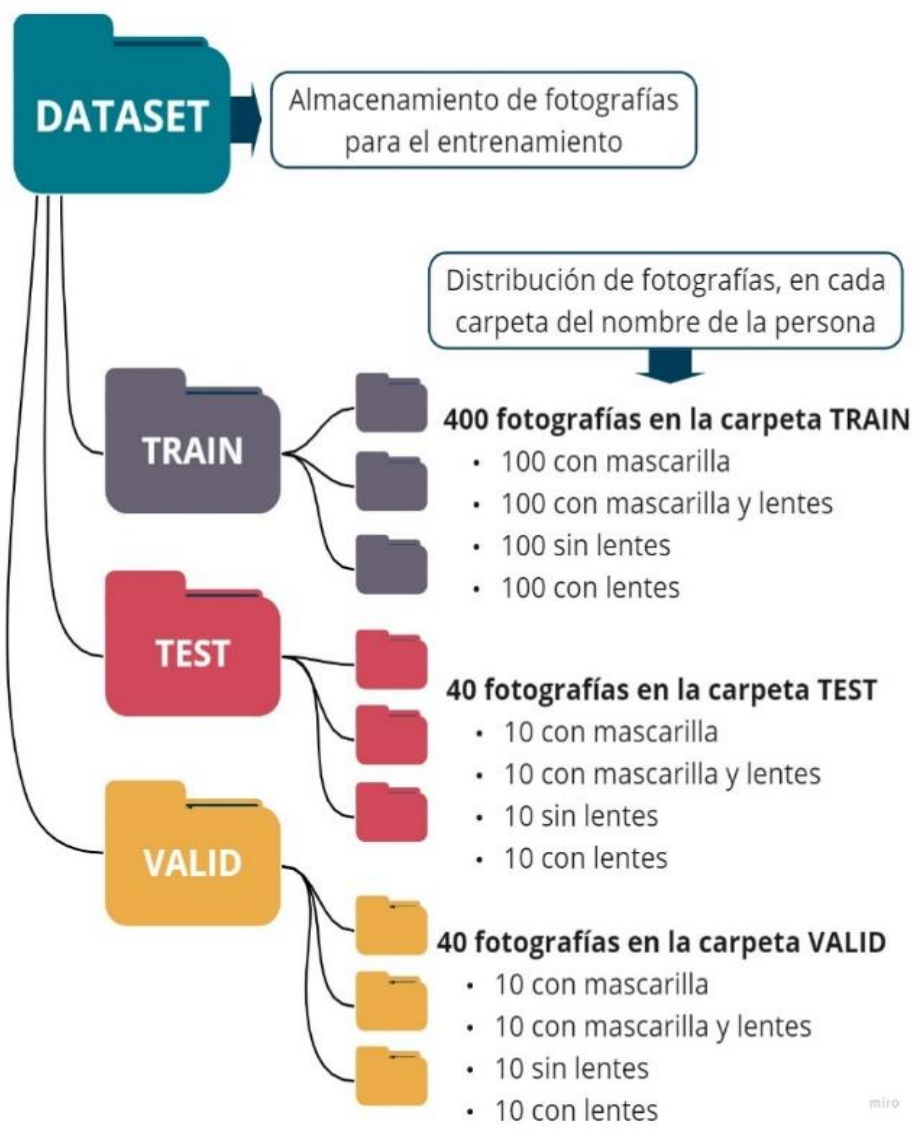

Figura 2: Distribución del dataset

Tal y como se muestra en la figura 3 , se da el proceso de facilitar la utilización del dataset para el entrenamiento de la red neuronal, que se realiza mediante la transferencia de aprendizaje de máquina, con el uso de la red pre entrenada VGG16, el cual facilita el procesamiento de las imágenes.

Se realizó el entrenamiento en 500 epochs, al finalizar el aprendizaje se obtuvo la red neuronal entrenada, la cual se descargó con el nombre "Red.h5".

\subsection{Etapa 3: ejecución}

Para el reconocimiento, previamente se aloja en google drive la red entrenada, y se sigue el mismo proceso de descomprimir el dataset.

La utilización de la librería Open CV y haardcascade frontalface es esencial, junto con las librerías Numpy y Phyton para la identificación del patrón de la imagen y sus diversas características usando el espacio vectorial para el reconocimiento en tiempo real al momento de la activación de la webcam.

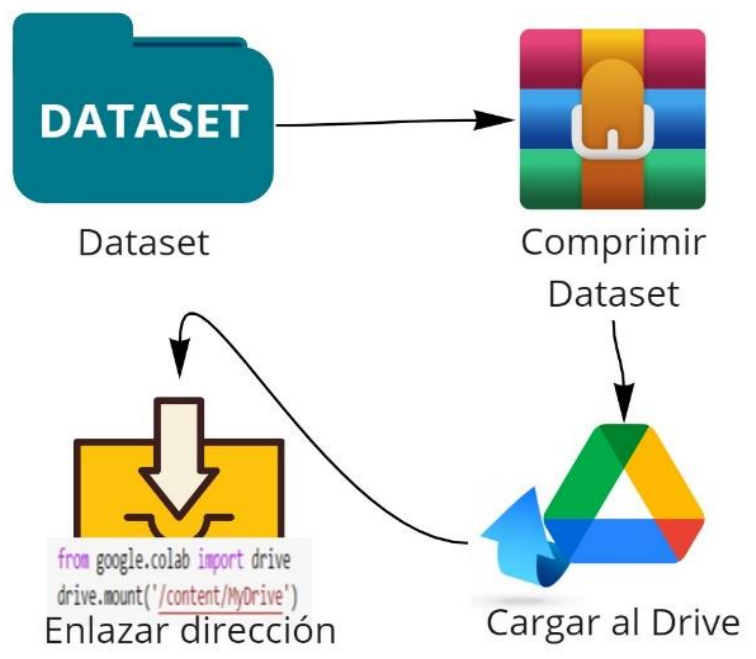

en el algoritmo

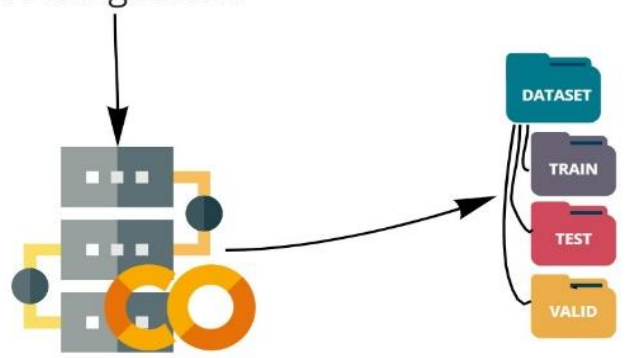

Ejecutar algoritmo

Dataset

para descomprimir

descomprimido

Figura 3: Descomprimir dataset 


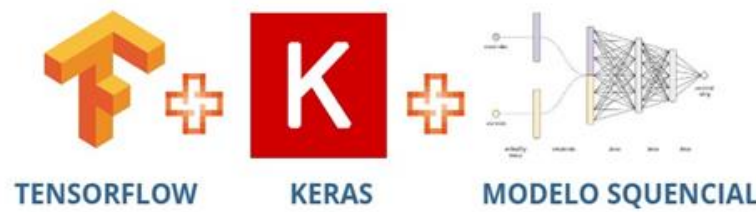

Importar librerias

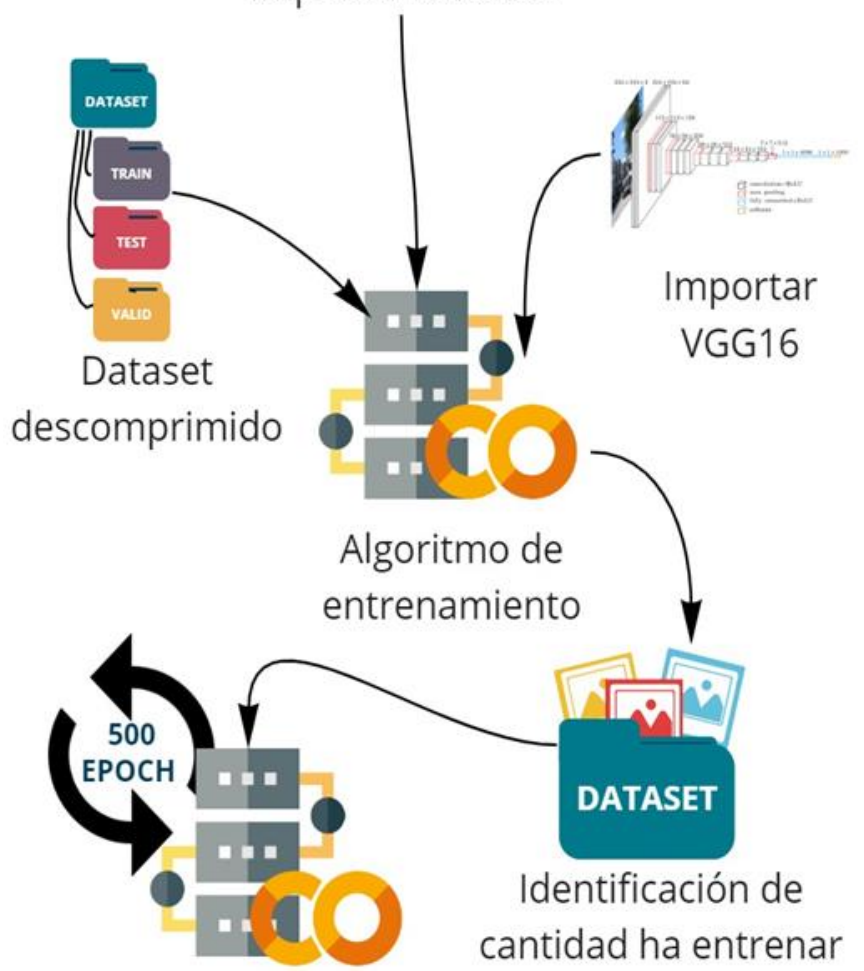

compilación de algoritmo

para el entrenamiento de

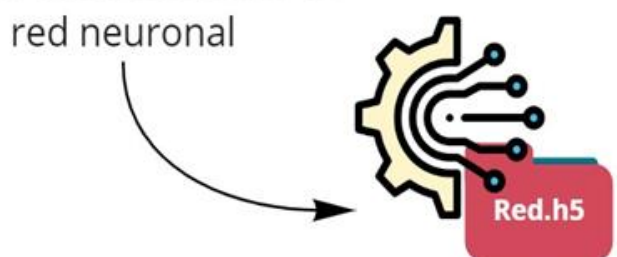

Red neuronal entrenada "Red.h5"

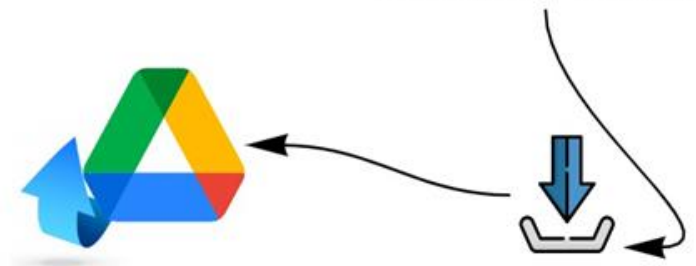

Cargar Red

neuronal a Drive

Descarga de Red neuronal

Figura 4: Entrenamiento de la red neuronal

\subsection{Etapa 4: análisis de resultados}

Realizando diferentes pruebas en relación a los tipos planteado que son rostro sin mascarilla y sin lentes, rostro con mascarilla sin lentes, rostro con lentes y mascarilla, rostro con lentes sin mascarilla, estas pruebas se clasifican por tipo para analizar el porcentaje de reconocimiento de cada uno de ellos, de manera que a través de la aplicación del Deep Learning que cumpla un porcentaje de precisión aceptable al reconocer e identificar el rostro de las personas, incluyendo las restricciones que sean causados por mascarillas o lentes que reduce los rasgos faciales para un mejor reconocimiento.

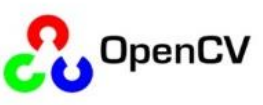

OPEN CV

\section{Importar librerias}

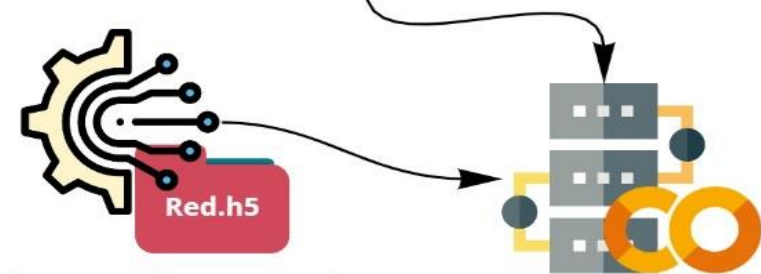

Enlazar red neuronal entrenada "Red.h5"

Algoritmo de activación de Webcam

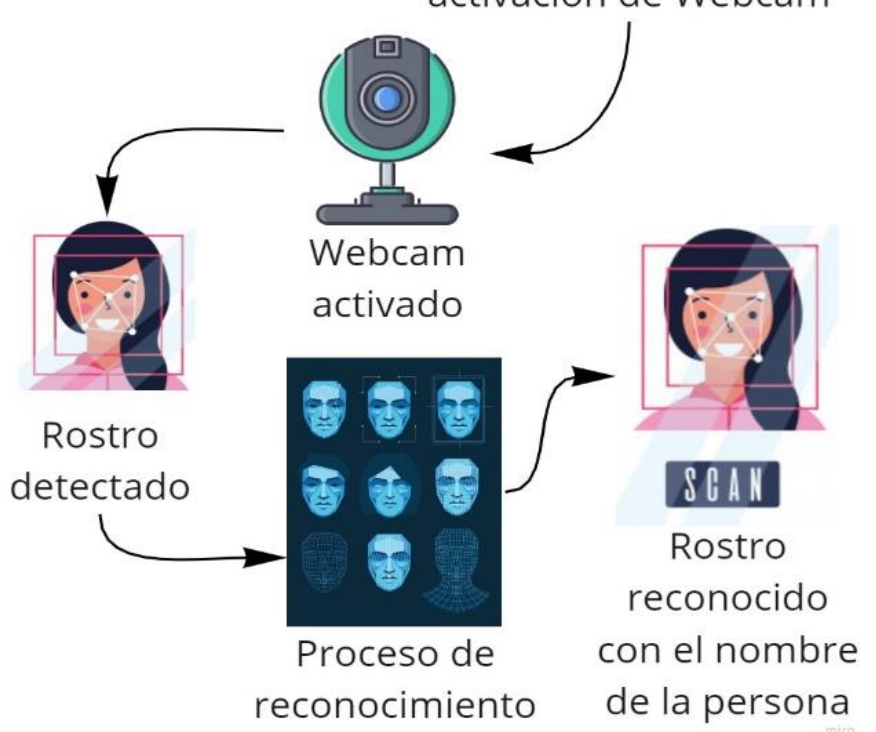

Figura 5: Reconocimiento facial

\section{Resultados y Discusión de resultados}




\subsection{Resultado de entrenamiento}

El entrenamiento se dio con 500 epochs o ciclos, los cual la red neuronal fue aprendiendo en un aproximado de 8 horas aproximadamente, dando un resultado de presición en el reconocimiento de 0.2 , el cual muestra una cierta aproximación a la presición exacta ,0.1. La presición muestra el número de errores que comete el modelo, después de comparar con el objetivo principal, esto se debe a la cantidad de epoch que se pone para el entrenamiento ,puesto que la muestra es de una amplia magnitud.Pero si se da el aumento, proporcionalmente se da el aumento de horas en el entrenamiento, puesto que influye maquinaria que se usa para realizar el entrenamiento.

\subsection{Resultado de reconocimiento}

Se realizó las pruebas de aplicación del entrenamiento, de un total de 5 personas como muestra, con un tiempo de 10 segundos de reconocimiento mediante la clasificación de los siguientes casos: sin lentes-sin mascarilla (SS), sin lentes-con mascarilla (SC), con lentes-sin mascarilla (CS) y con lentes-con mascarilla (CC), tabla 1.

Tabla 1: Prueba por clases y tipos

\begin{tabular}{|c|c|c|c|c|c|}
\hline \multirow{2}{*}{ CLASE } & \multicolumn{4}{|c|}{ TIPOS } & \multirow{2}{*}{ PORCENTAJE } \\
\cline { 2 - 5 } & SS & SC & CS & CC & \\
\hline $\begin{array}{c}\text { Roy } \\
\text { Uscamayta }\end{array}$ & $100 \%$ & $90 \%$ & $80 \%$ & $60 \%$ & $82.5 \%$ \\
\hline $\begin{array}{c}\text { Jemima } \\
\text { Elias }\end{array}$ & $100 \%$ & $80 \%$ & $100 \%$ & $70 \%$ & $87.5 \%$ \\
\hline $\begin{array}{c}\text { Angiela } \\
\text { Rojas }\end{array}$ & $100 \%$ & $80 \%$ & $70 \%$ & $40 \%$ & $72.5 \%$ \\
\hline $\begin{array}{c}\text { Milagros } \\
\text { Alfaro }\end{array}$ & $40 \%$ & $40 \%$ & $30 \%$ & $20 \%$ & $32.5 \%$ \\
\hline $\begin{array}{c}\text { Luis } \\
\text { Salazar }\end{array}$ & $90 \%$ & $80 \%$ & $80 \%$ & $70 \%$ & $80 \%$ \\
\hline \multicolumn{4}{|c|}{ PORCENTAJE TOTAL } & $71 \%$ \\
\hline
\end{tabular}

Se obtuvo como resultado de las pruebas la precisión del reconocimiento facial de 4 personas de un total de 5 , lo cual demuestra lo nombrado de los resultados del entrenamiento, dando un porcentaje total aprobado en contraste al objetivo trazado.
Tabla 2. Prueba Roy Uscamayta

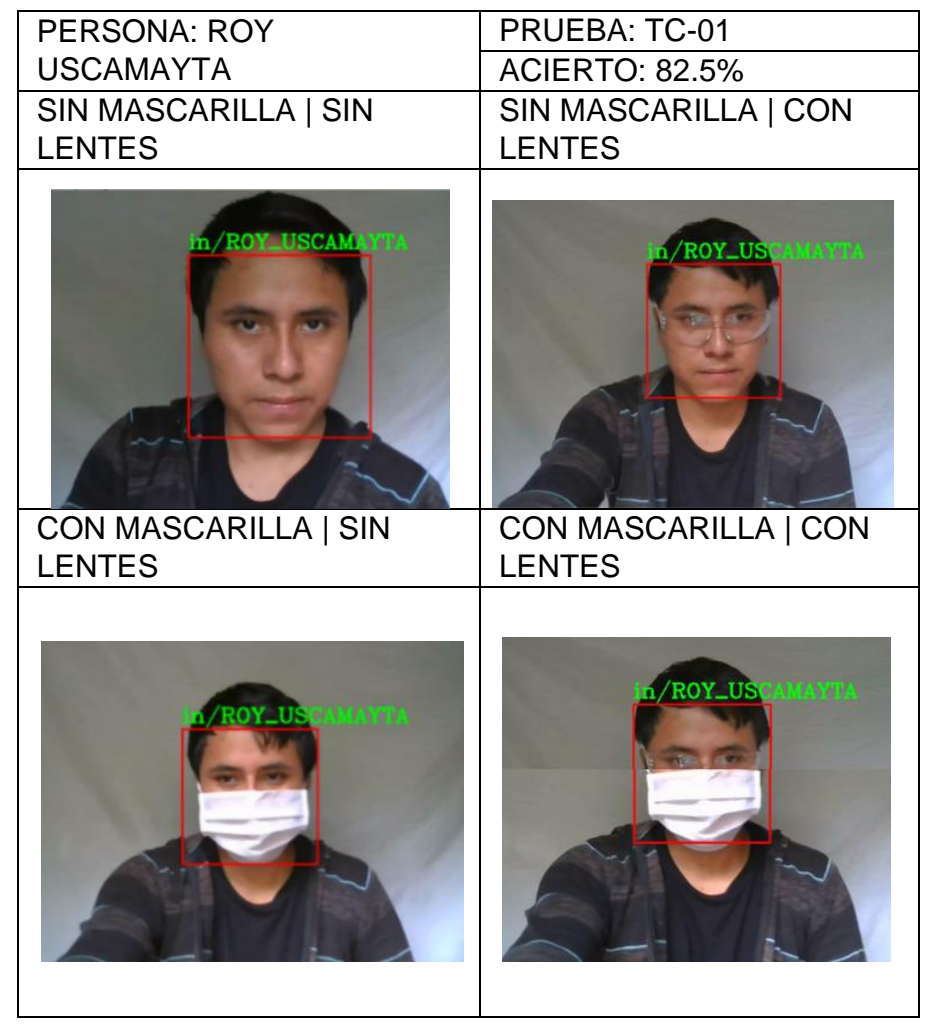

Tabla 3. Prueba Jemima Elias

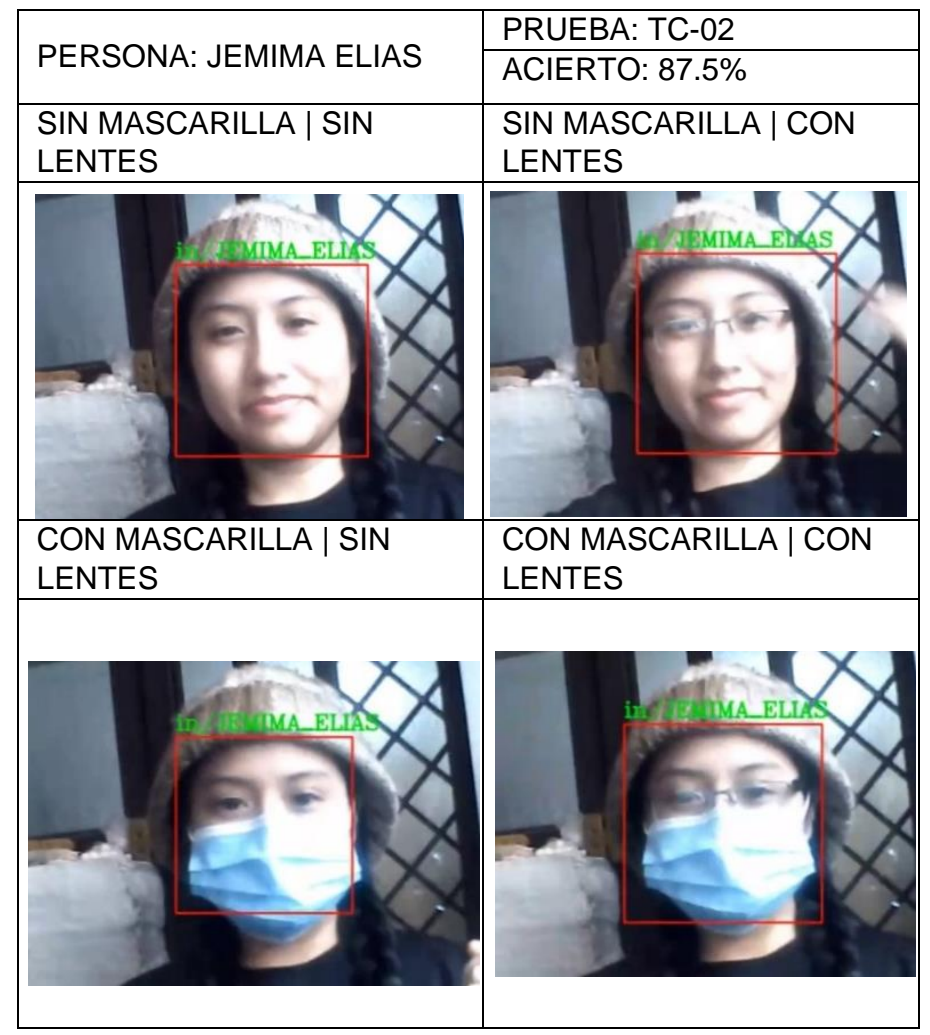


Tabla 4. Prueba Milagros Alfaro

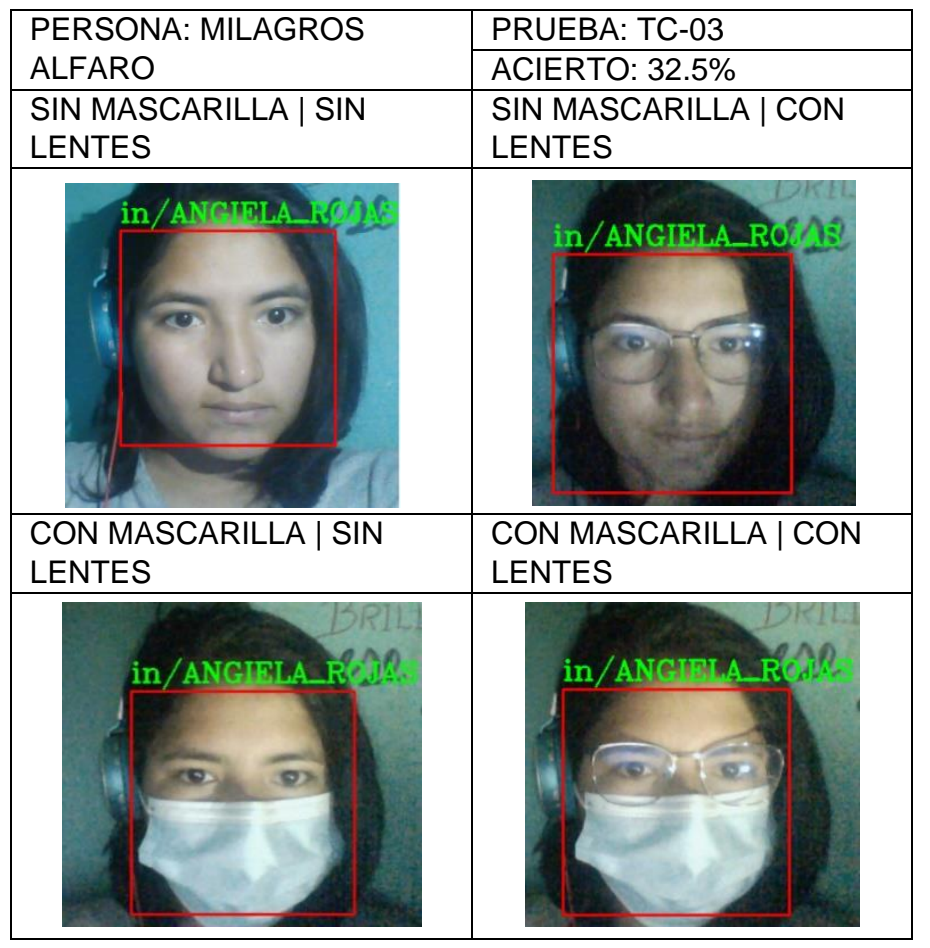

Tabla 5. Prueba Angiela Rojas

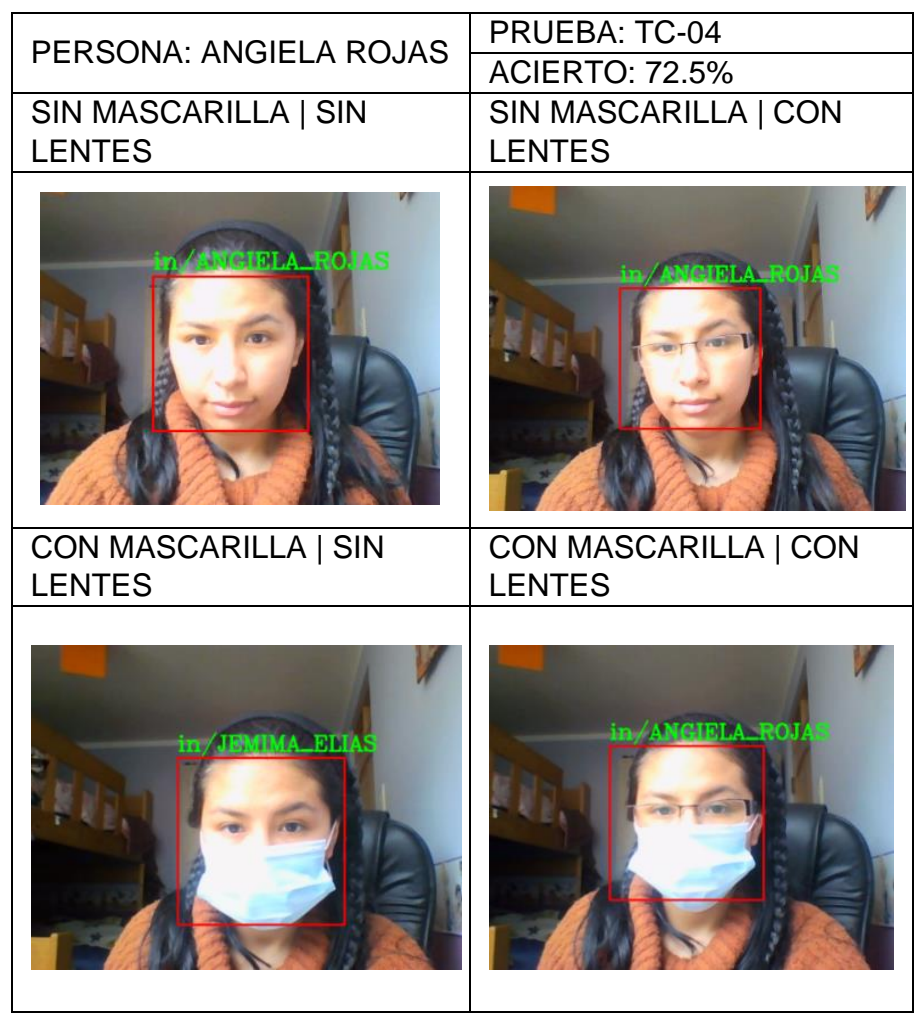

Tabla 6. Prueba Luis Rojas

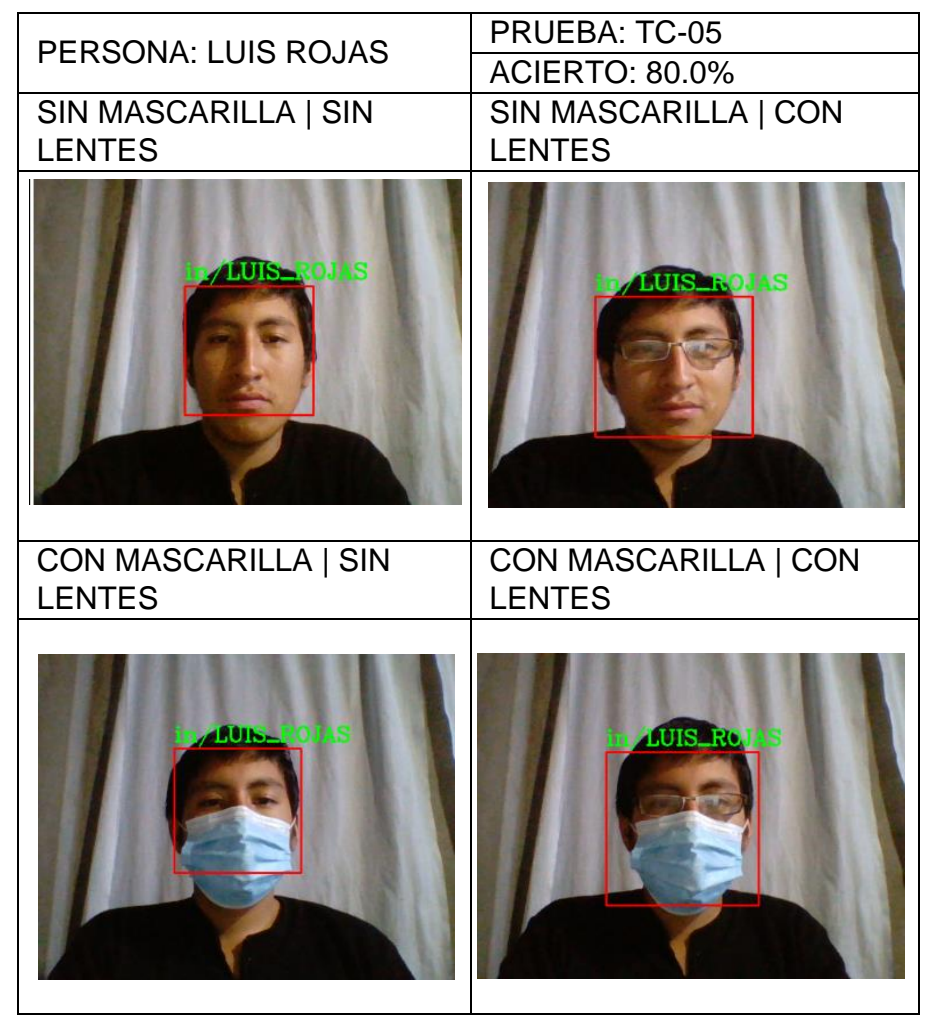

El reconocimiento de cada muestra de estudio se observa en la tabla 2., tabla 3 , tabla 4 , tabla 5 y tabla 6 , describiendo el acierto y las pruebas de reconocimiento según la clasificación de cada caso.

\section{Conclusiones}

El porcentaje de precisión alcanzado en la fase de entrenamiento de la red neuronal es de 0.2 , la cual demuestra el $20 \%$ de error en la fase de reconocimiento, obteniendo así un $71 \%$ de acierto en la realización de las pruebas, observándose un buen resultado con respecto al porcentaje de error en el reconocimiento de la persona utilizando mascarilla, lentes, incluso si la persona está usando ambos accesorios o no, dado que la red neuronal creada permite el reconocimiento facial con presencia de oclusiones como lo son los lentes y la mascarilla.

El uso de librería de VGG16 permite la transferencia de aprendizaje en el entrenamiento y el fácil procesamiento de las imágenes que se encuentran en el dataset. 
El tiempo de demora del entrenamiento de la red neuronal a 500 epoch es de aproximadamente 8 horas en google colab, trabajando con 2400 imágenes, por lo que el tiempo de procesamiento depende de la cantidad de imágenes a entrenar y de los ciclos de entrenamiento.

Los porcentajes de reconocimiento vistos en las pruebas de eficacia dependen mucho de las fotos de entrenamiento que se realiza, entre más fotos y más sea los movimientos de la rotación de la cabeza se capturen, será mejor el reconocimiento facial con el uso de mascarilla, lentes, ambos accesorios a la vez o con ninguno de ellos.

\section{Agradecimiento}

Agradecemos primeramente a Dios, a nuestros padres que cada día nos dan su apoyo y a la Universidad Nacional del Centro del Perú por la oportunidad y apoyo hacia nuestra persona.

\section{Referencias}

[1] H. Yang y X. Han, «Face Recognition Attendance System Based on Real-Time Video Processing", IEEE Access, vol. 8, pp. 159143-159150, 2020, doi: 10.1109/ACCESS.2020.3007205.

[2] «El reconocimiento facial se abre paso como opción segura para registrar ingresos a los trabajos». https://elcomercio.pe/videos/tecno/elreconocimiento-facial-se-abre-paso-comoopcion-segura-para-registrar-ingresos-a-lostrabajos-video-nnav-agefe-tecno-noticia/ (accedido jun. 20, 2021).

[3] L. Xu, L. Huang, y C. Liu, «Raw vs. Processed: How to Use the Raw and Processed Images for Robust Face Recognition under Varying Illumination», en 2010 20th International Conference on Pattern Recognition, ago. 2010, pp. 2692-2695. doi: 10.1109/ICPR.2010.660.

[4] F. D. Guillén-GáMez, I. García-Magariño, J. Bravo-Agapito, R. Lacuesta, y J. Lloret, «A proposal to improve the authentication process in m-health environments", IEEE Access, vol. 5, pp. 22530-22544, 2017, doi: 10.1109/ACCESS.2017.2752176.

[5] M. Chihaoui, A. Elkefi, W. Bellil, y C. Ben Amar, «A Survey of 2D Face Recognition Techniques»,

Computers, vol. 5, n. ${ }^{\circ} 4$, Art. n. ${ }^{\circ}$ 4, dic. 2016, doi: $10.3390 /$ computers 5040021.

[6] "COVID-19: cronología de la actuación de la OMS». https://www.who.int/es/news/item/27-042020-who-timeline---covid-19 (accedido jun. 20, 2021).

[7] El Peruano, «Decreto Supremo No 184-2020PCM », Decreto Supremo que modifica el numeral 8.4 del artículo 8, el artículo 9 y el numeral 14.2 del artículo 14 del Decreto Supremo $N^{\circ} 184-2020-$ PCM.

https://busquedas.elperuano.pe/normaslegales/d ecreto-supremo-que-modifica-el-numeral-84-delarticulo-8-decreto-supremo-n-083-2021-pcm1947038-1/ (accedido jun. 20, 2021).

[8] B. López Takeyas, Introducción a la Ingeniería en Sistemas Computacionales y al Diseño Orientado a Objetos. Pearson, 2019. Accedido: jun. 20, 2021. [En línea]. Disponible en: http://www.itnuevolaredo.edu.mx/takeyas/LibrolS C/index.htm

[9] Á. Artola Moreno, «Clasificación de imágenes usando redes neuronales convolucionales en Python», Universidad de Sevilla, 2019. [En línea]. Disponible en: http://bibing.us.es/proyectos/abreproy/92402/fich ero/TFG-2402-ARTOLA.pdf

[10] I. Masi, Y. Wu, T. Hassner, y P. Natarajan, «Deep Face Recognition: a Survey», p. 8.

[11]«IBM mejora el reconocimiento facial con aprendizaje profundo", Máster en Deep Learning: Universidad de Alcalá, jul. 10, 2019. https://master-deeplearning.com/ibmreconocimiento-facial-aprendizaje-profundo/ (accedido jun. 20, 2021).

[12]J. Liu, H. Wang, y Y. Feng, «An End-to-End Deep Model With Discriminative Facial Features for Facial Expression Recognition», IEEE Access, vol. 9, pp. 12158-12166, 2021, doi: 10.1109/ACCESS.2021.3051403.

E-mail:

e 20171005621@uncp.edu.pe
e 2017100580G@uncp.edu.pe
e 2017100581F@uncp.edu.pe
e 2017100553J@uncp.edu.pe
aegamarra@uncp.edu.pe

